\title{
Multi-Grain Reconfiguration for Advanced Adaptivity in Cyber-Physical Systems
}

\author{
Tiziana Fanni*, Alfonso Rodríguez ${ }^{\dagger}$, Carlo Sau*, Leonardo Suriano ${ }^{\dagger}$, Francesca Palumbo ${ }^{\ddagger}$, \\ Luigi Raffo*, Eduardo de la Torre ${ }^{\dagger}$ \\ *DIEE, Università degli Studi di Cagliari; Email: tiziana.fanni@diee.unica.it \\ †Centro de Electrónica Industrial, Universidad Politécnica de Madrid; Email: alfonso.rodriguezm@upm.es \\ IDEA Lab, Università degli Studi di Sassari; Email: fpalumbo@uniss.it
}

\begin{abstract}
The advent of Cyber-Physical Systems (CPS) is putting designers to the test: during operation, such a kind of systems has to meet multiple and variable requirements coming from the environment, the user or the system itself. Runtime adaptivity allows addressing mutable requirements over time by modifying system behavior, and it can be implemented in hardware leveraging on reconfigurable devices operating at different granularities: mainly coarse- and fine-grain. The former is faster but less flexible (minor modifications are applied) than the latter. For modern CPS an advanced adaptivity support providing both the advantages of the two main hardware reconfiguration approaches could be extremely useful. In this work, a novel approach for multi-grain reconfiguration is presented in order to deliver advanced adaptive hardware accelerators to CPS.

Index Terms-Cyber-Physical Systems, Hardware Reconfiguration, Adaptive Systems
\end{abstract}

\section{INTRODUCTION}

Cyber-Physical Systems (CPS) are complex systems, characterized by a strong entanglement with the environment and integrating a plethora of different interacting components. These latter should be potentially capable of supporting multiple and distinct behavioral modalities to satisfy adaptation when needed. Both environmental (captured through sensors) and system (captured through monitors) awareness are meant to allow prompt reaction to changes. CPS have to guarantee varying workloads and performance objectives, while contin uously optimizing performance goals, i.e. minimizing energy consumption, meeting the available power budget, and so on.

To address adaptivity, computing layers need to be flexible, which may be colliding with performance guarantees. General purpose (i.e. CPUs, GPUs and DSPs) substrates are capable of implementing any application, but they are not specialized, resulting unable to offer high execution efficiency. Application Specific Integrated Circuits (ASICs) boost performance, being customized on the application they are designed for, but they are not flexible. In between, an appealing solution is provided by reconfigurable approaches. They are generally composed by a network of configurable processing elements (PEs), that can compute simple or more complex functions, whose interconnections are also configurable. According to their granularity, reconfigurable systems can be divided in: Fine-Grain (FG) and Coarse-Grain (CG).

FG reconfigurable systems apply reconfiguration at the single bit level, thus offering high flexibility. However, they involve a large amount of PEs and lots of interconnect points to be configured, so that the configuration bitstream size is big (usually a configuration memory is required instead of few configuration registers) and the related reconfiguration time is long. The most common example of FG architectures are FPGAs.Recently, Dynamic Partial Reconfiguration (DPR) has been proposed to mitigate those drawbacks by limiting the area involved, making it possible to perform it also at runtime. DPR systems usually have a static region that never changes during execution and one or more Reconfigurable Partitions (RP), where different circuits can be allocated at runtime [1]. DPR systems can switch among functionalities: one single highly specialized accelerator is implemented in the FPGA at a time, while the others are stored in a memory and can be implemented via reconfiguration. The deployed accelerator is always optimized in terms of resource usage and frequency, but reconfiguration requires a certain amount of time and an associated power consumption (proportional to the RP size) [2]. DPR-capable floorplans usually involve coarse regions of the FPGA. In the rest of this paper, the adopted terminology refers only to the granularity on which reconfiguration is applied (bit or word level): DPR/FR versus the specific CG presented.

CG reconfigurable (CGR) approaches demonstrated to be a viable solution to provide adaptivity in CPS, trading off functional and non-functional requirements [3]. Contrarily to FG/DPR architectures, CGR engines apply reconfiguration at the word/data level. Computing and interconnecting resources are configured word by word, rather than considering single bits; therefore, reconfiguration can be performed quickly due to the limited configuration stream size, which in turn means less memory to store it [4]. Word level reconfigurability limits flexibility: these systems are able to switch among a limited set of kernels. From the implementation point of view, CGR is virtual: all the resources are available in the substrate, being multiplexed in time while switching among configurations. This affects both operating frequency (multiplexers make the critical path longer) and resource usage (the PEs for all the kernels are always on chip). Commonly, designers use a hardware-to-application approach: a generic CGR structure of homogeneous or heterogeneous PEs with a (potentially) configurable interconnect is made available for mapping different kernels. Such an approach favors flexibility at the price of 
the execution efficiency of the single applications [5], [6]. To improve efficiency, the opposite approach (i.e., application-tohardware) can be used. In this case, the CGR structure involves specialized PEs for the execution of the applications it has been conceived for, but it is not possible to add additional applications afterwards [7].

This works combines the two main hardware reconfiguration approaches, namely FG and CG, to deliver the best of both and better cope with the challenging CPS adaptivity needs.To this end, an automated toolchain has been assembled to foster productivity. The toolchain goes from high-level dataflow specifications of the desired applications (modeling them is the unique effort required to the user) to the FPGA implementation of multi-grain adaptive systems, where different RP of an FPGA are filled in with heterogeneous and irregular application-specific CGR datapaths. The toolchain provides both design-time and run-time support by automating the system generation and its management. As a first proof of concept of the multi-grain reconfigurable architectures generated by the toolchain, a use-case involving two edge detection kernels has been adopted. Experimental results show the potentials of the approach when adaptivity needs change: sometimes a low overhead slight adjustment of the computation through CGR can be enough (e.g. to modify the implemented detector), while other times a strong modification of the system behavior by means of DPR is mandatory (e.g. to maximize execution efficiency when the image size changes).

The rest of this paper is organized as follow: Section II provides background and related works. Section III describes the proposed integrated design flow for multi-grain reconfiguration. Section IV presents assessment results on a use-case exploiting two different algorithms for edge detection.

\section{RELATED WORK AND BACKGROUND}

This section provides a small overview of state of the art approaches for multi-grain adaptivity (see Section II-A), highlighting what is already available and what is done differently in this work. Moreover, a description of the bases for the proposed architecture and toolchain is also presented (see Section II-B and Section II-C).

\section{A. Literature on Multi-Grain Adaptivity}

Different works explored the adoption of both FG and CG reconfigurability on the same substrate to combine their benefits together. Modern FPGAs themselves are actually multi-grain platforms, since they include CG reconfigurable blocks, as BRAMs and DSPs, spread within an FG substrate. Amagasaki et al. [8] proposed the Variable Grain Logic Cell (VGLC) architecture that adjusts computational granularity according to the application. It adopts virtual reconfiguration, leveraging on four configurable units that perform operations such as arithmetic logic, random logic, and multiplexing in any type of application. The HoneyComb architecture [9] is an adaptable dynamically reconfigurable cell array. Cells are composed of a routing and a computing part. Routing parts, responsible for connecting neighbors, compose the reconfigurable interconnection network. Functional parts can be enabled, disabled, or modified using DPR. The DeSyRe SoC [10] leverages on a multi-grain texture containing different sub-components surrounded by reconfigurable interconnects. Along with functional adaptivity, it also supports repairoriented one by providing fault tolerance with re-routing, retargeting functionalities on unused sub-components, or by a functionally-equivalent instance implemented in FG reconfigurable hardware. Yuan et al. [11] presented a multi-grain FPGA aimed for mobile computing and focused on two key steps towards higher efficiency: interconnection network and CG reconfigurable digital signal processors. The chip incorporates FG configurable logic blocks, medium-grain digital signal processors with reconfigurable block RAMs, and two CG kernels.

All the above-mentioned approaches are limited to partially reconfigurable CG arrays, where the PEs are mostly homogeneous and not directly derived from the applications to be accelerated. This lack of specialization may limit performance, which is made even worse if infrastructures with fixed interconnection are used. This work proposes a multi-grain framework flexible enough to be suitable to support functional and non-functional adaptivity. Moreover, with respect to the above-mentioned works in literature, it not only presents a novel architecture, but it also delivers a complete toolchain where user has only to model the desired applications in a high-level dataflow language, while the design and run-time management of the architecture is automated.

\section{B. How to build DPR overlays: the ARTICo ${ }^{3}$ Architecture}

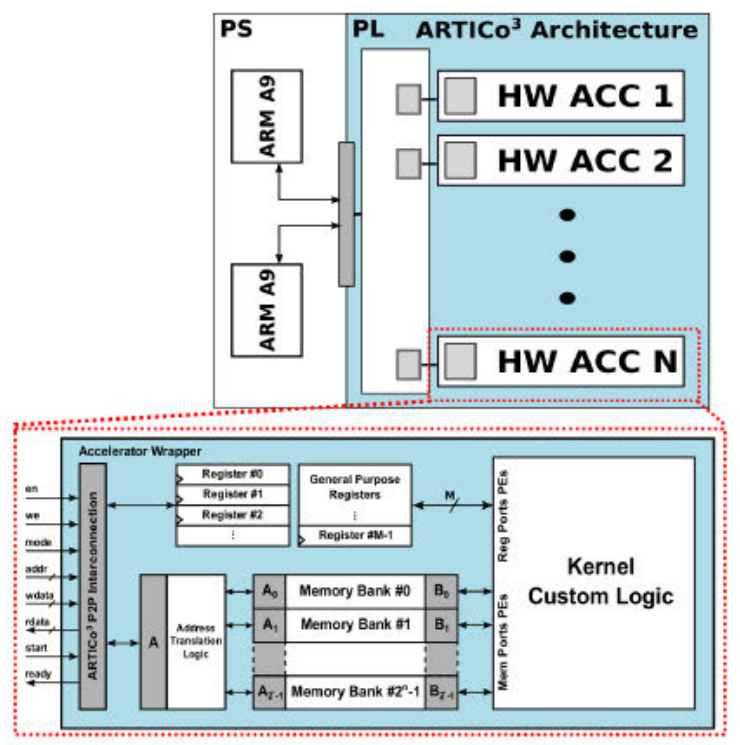

Fig. 1. Schematic view of the ARTICo ${ }^{3}$ Architecture.

The use of SRAM-based FPGAs has merged the best of two worlds (i.e. hardware and software), enabling systems with software-like flexibility while keeping high-performance benefits of dedicated hardware-based processing. The specific 
technology that supports this feature is the DPR. The ARTICo ${ }^{3}$ architecture exploits DPR in high-performance embedded systems that use a processor-coprocessor approach [12]. However, instead of relying only on one application-specific hardware accelerator for each task, as it has been traditionally done, the computing fabric supports a multi-accelerator based computing scheme. Similarly to embedded GPUs supporting general purpose computing, the $\mathrm{ARTICo}^{3}$ computing fabric usually operates in SIMD-like fashion (Single Instruction Multiple Data), where different copies of a given hardware accelerator work on different sets of input data.

ARTICo $^{3}$-based hardware accelerators are connected to the communication infrastructure using a custom gateway able to dynamically alter its internal datapath to meet specific requirements of computing performance or energy consumption. The gateway hides custom point-to-point interfaces behind a standard AXI4 interface. Plug-and-play capabilities are enabled in user-defined custom accelerators by instantiating them in wrapper modules embedding a local memory (divided in banks) and a register bank (for configuration purposes). Figure 1 depicts a schematic view of the $\mathrm{ARTICo}^{3}$ architecture with a zoom on the ARTICo ${ }^{3}$ slot wrapper. The ARTICo ${ }^{3}$ computing fabric is part of a complete framework able to automatically build the fabric starting from either $\mathrm{C} / \mathrm{C}++$ or HDL application specifications (custom accelerators), and manage application execution and computation offloading to the hardware accelerators using its Runtime Library. For further details on ARTICo ${ }^{3}$ computing fabric implementation and management please see [12].

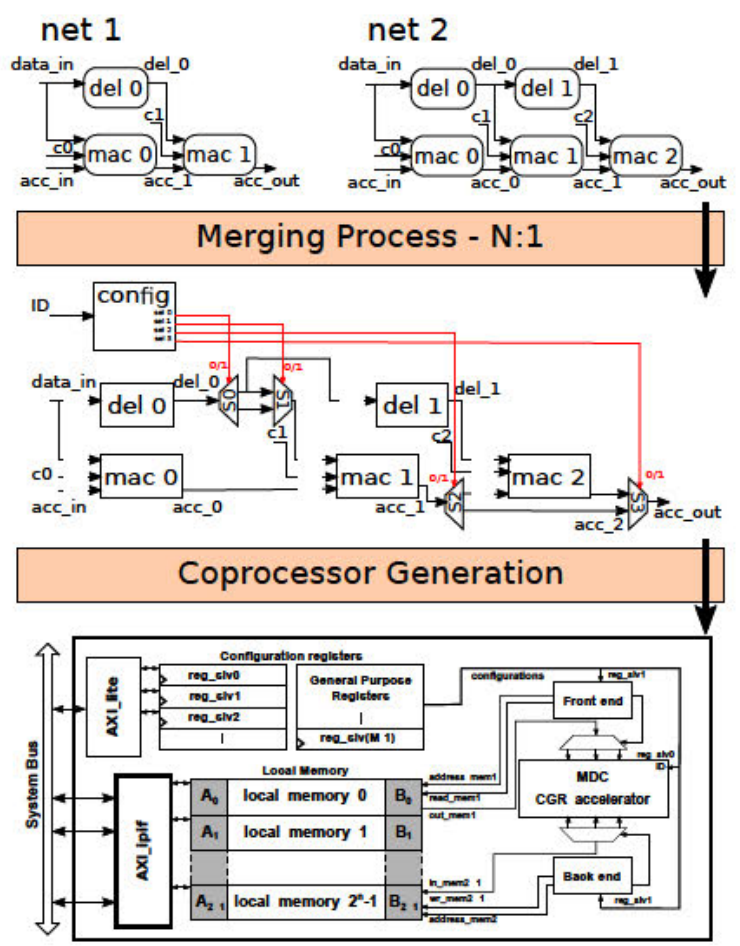

Fig. 2. MDC Design Flow
C. How to implement application specific CGR datapaths: The Multi-Dataflow Composer

The Multi-Dataflow Composer (MDC) is an automated framework that, through an application-to-hardware approach, generates heterogeneous and irregular CGR substrates. Applications to be implemented are described as dataflow models [13] and combined together through an iterative datapathmerging algorithm, which shares dataflow actors (PEs) among the different application models, leading to the composition of an irregular computing core containing only the logic and connections necessary to guarantee the correctness of the input applications. The generated CGR accelerator is heterogeneous, PEs are actor-specific rather than homogeneous, and may be manually written or synthesized from the actors by using highlevel synthesis techniques [14]. To access shared resources multiplexers, named Switching-Boxes (SBoxes), are inserted in the accelerator. Once all the input dataflow models are combined together, MDC generates the corresponding HDL. The user has only to model applications as dataflows, while MDC takes care of automatically generating the corresponding CGR accelerators. Figure 2 depicts an example of the MDC operation with 2 input dataflows whose combination requires the insertion of 4 SBoxes, for a resulting configuration pattern size of 4 bits.

MDC offers also the possibility of seamlessly integrating the CGR logic as an adaptive hardware accelerator in a processorcoprocessor system for Xilinx environments [15]. By analyzing the features of the combined dataflow specification suitable wrappers and APIs for different processor-coprocessor communication infrastructures (memory mapped or stream) are automatically provided. Bottom part of Figure 2 shows a scheme of the Xilinx IP generated by MDC.

\section{Toolchain For Multi-grain Reconfiguration}

As explained in Section II-B, the ARTICo ${ }^{3}$ architecture offers high flexibility, operating in SIMD-like fashion. However, this flexibility is obtained paying the cost of DPR reconfiguration, that requires long time and high energy consumption. When frequent changes among functionalities are required, this cost may easily become no longer affordable. On the other hand, the MDC Tool provides N:1 composition/synthesis. As described in Section II-C, MDC automatically deploys a heterogeneous and irregular CGR accelerator starting from an input set of dataflow specifications. CGR accelerators generated by MDC offer high-speed low-energy reconfiguration, since here reconfiguration is performed by simply changing the application ID written on a configuration register. However, they are able to implement only the functionalities they have been designed for. Also, being a fixed design, the only way to provide parallelism is instantiating more accelerators in parallel, thus paying the cost of the extra logic if, during computation, parallelism is not required anymore.

Thus, ARTICo ${ }^{3}$ and the MDC flows clearly show opposite tradeoffs between flexibility and reconfiguration costs when considered individually. A combination of these two approaches would enable adaptive multi-grain reconfigurable 


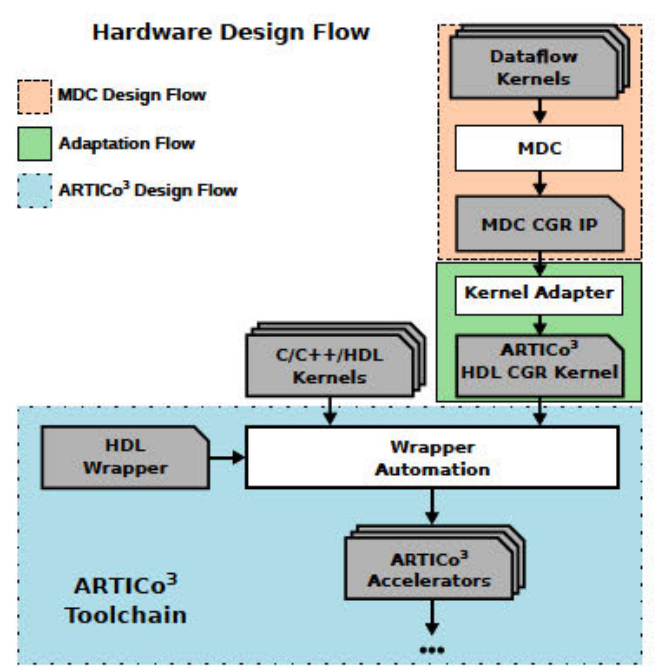

Fig. 3. Integrated Hardware Design Flow

fabrics exhibiting the benefits of both DPR and CGR. The result is a flexible advanced hardware adaptivity which can meet the changing functional and non-functional requirements of CPS designs. In particular, users would have the flexibility to adopt CGR when the application requires frequent switching between graphs/functionalities with shared resources, while DPR can be used either to change the level of parallelism or to change completetly the computing core, when the instantied CGR logic is no longer required.

In this work, the ARTICo ${ }^{3}$ framework and the MDC design flow have been combined in a unique toolchain, capable of automatically implementing and managing multi-grain reconfigurable systems. This flow, starting from the high-level dataflow descriptions of the applications to be accelerated within the configurable logic, derives the multi-grain reconfigurable substrate, placing CGR MDC-compliant datapaths within the DPR slots of the ARTICo ${ }^{3}$ architecture as depicted in Figure 3. With respect to the standalone flows, an adaptation step (Kernel Adapter) is needed. First, user-driven dataflow specifications are processed by MDC to generate a CGR IP. This IP is modified by the Kernel Adapter, which delivers an HDL ARTICo ${ }^{3}$-compliant CGR kernel. Finally, the ARTICo ${ }^{3}$ framework processes the input HDL CGR kernel to implement the whole reconfigurable processing system.

The ARTICo $^{3}$ toolchain already takes care of adding the necessary glue logic to interface each hardware accelerator (located in RP) with the static region. Thus, it requires as input only the core logic of the accelerator. Figure 4 shows an example where an MDC-generated IP, with one input port and one output port, requiring three configuration registers and two memory blocks, is modified through the Adaptation Flow. The Adaptation Flow parses the MDC CGR IP to remove all interfacing logic (AXI-lite interface, AXI-full interface, local memories and configuration register bank). The remaining logic is instantiated in an HDL wrapper whose external interface is the one required in a standalone $\mathrm{ARTICo}^{3}$ kernel specification. During this step, the Adaptation Flow takes also

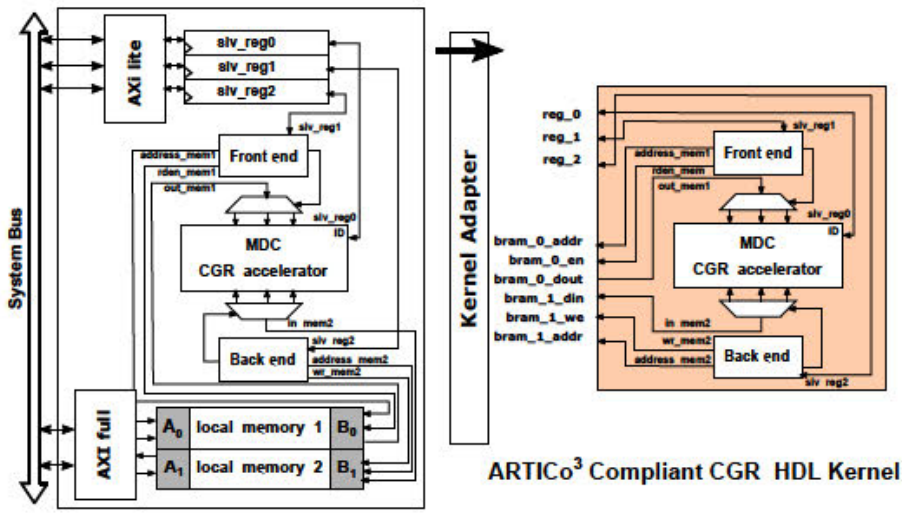

Fig. 4. Adaptation Flow from an MDC- to a ARTICo ${ }^{3}$-compliant CGR IP.

care of properly connecting the ports of the new HDL wrapper to the internal CGR-kernel logic.

\section{AsSESSMENT}

In this Section, as a proof of concept, the proposed multigrain reconfiguration approach is evaluated through an edge detection application involving two different algorithms: Sobel and Roberts. This use-case enables the possibility of evaluating the advanced adaptivity features regarding functional (changing the edge detection algorithm) and non-functional (tradeoff in terms of execution time and the power) requirements, acting as a prototype example of CPS behavior. Assessment results have been collected using a custom Zynq-7000 board, based on the XC7Z020CLG484-1 device, with integrated power monitoring circuitry.

\section{A. Test Case: Edge Detection - Sobel and Roberts algorithms}

Edge detection algorithms estimate the magnitude and the orientation of edges on a greyscale image [16]. They are adopted in several application fields, such as image segmentation, image compression, computer vision and security. Edge detectors are usually computationally intensive tasks that are accelerated in hardware. The simplest techniques involve discrete first-order differentiation operators. Indeed, the boundary of an object is the difference of the intensity levels in its pixels with respect to the surrounding pixels. To assess the proposed architecture, Sobel and Roberts detectors have been used. These operators are applied to evaluate the gradient image $G=k * A$, given by the convolution of the kernel $k$ with the source image $A$ ( $k$ is $3 \times 3$ for Sobel and $2 \times 2$ for Roberts).

The $\mathrm{G}$ function, corresponding to the magnitude of the edge, is calculated as $G=\sqrt{G_{x}^{2}+G_{y}^{2}}$, where $G_{x}$ and $G_{y}$ are obtained as:

$$
G_{x}=\left[\begin{array}{ccc}
1 & 0 & -1 \\
2 & 0 & -2 \\
1 & 0 & -1
\end{array}\right] * A \quad, \quad G_{y}=\left[\begin{array}{ccc}
1 & 2 & 1 \\
0 & 0 & 0 \\
-1 & -2 & -1
\end{array}\right] * A
$$

for the Sobel case [17], and as:

$$
G_{x}=\left[\begin{array}{cc}
1 & 0 \\
0 & -1
\end{array}\right] * A \quad, \quad G_{y}=\left[\begin{array}{cc}
0 & 1 \\
-1 & 0
\end{array}\right] * A
$$


for the Roberts case [18]. The magnitude of the edge, $G$, can be approximated as $\left(\left|G_{x}\right|+\left|G_{y}\right|\right) / 2^{n}$, where $n$ is a scaling factor. As soon as the magnitude is evaluated, a thresholding stage (the threshold value depends on the specific context of application) compares the magnitude with a desired value, to determine whether the edge is present or not.

The Roberts edge detector is the simplest gradient-based edge detector, due to the reduced convolution kernel matrix. It requires a bit less computation, resulting in limited footprint when implemented in hardware, with respect to Sobel [19]. However, this simplicity is paid in terms of detection effectiveness and noise robustness: Roberts is able to detect a smaller set of edges [20], and it is more sensitive to noise than Sobel [21]. Due to these characteristics, implementing both Sobel and Roberts edge detectors on the same device can be useful to achieve non-functional adaptivity. Sobel and Roberts kernels may correspond to different working points, each featuring a different tradeoff in terms of performance and detection power: Roberts constitutes the fastest but less accurate detection, while Sobel pushes detection quality at the price of a slower execution. Such kind of changing behaviors could be suitable for CPS contexts, where the system has to monitor the environment, the user demand and its internal state to promptly adapt the exhibited behavior. For example, if the battery level of the system is lower than a certain threshold, it can switch from a more consuming detector (Sobel) to a less power-hungry one (Roberts). In the same way, if the incoming images are not so noisy, a low quality detector (Roberts) can be sufficient and more energy efficient, while, if the image source is noisy, Sobel is preferable. Note that, such approach can also be exploited to achieve functional adaptation, when Sobel and Roberts have to be adopted in the same application, such as for airport runway tracking on infra-red images [22].

\section{B. Designs Under Tests}

As described in Section III, in order to foster design automation and ease the development of hardware accelerators, in the proposed design flow dataflow models are adopted for the specification of applications behavior. In this use-case, dataflow models have been described in CAPH language [23].

Figure 5 depicts simplified graphs of the dataflows representing Sobel and Roberts kernels, according to the algorithm described in Section IV-A. In particular, line buffer actors are adopted to store previous rows of the image, while delay actors are in charge of memorizing one previous pixel within a row. In such a way, once these actors are filled with proper numbers of rows and pixels, the convolution actors can start computing the horizontal and vertical gradients. Actor abs sum finalizes the magnitude computation step by summing up the absolute values of the horizontal and vertical gradients and by rightshifting the result for a given scaling factor $n$. Lastly, the thresholding actor $t h r$ sets to 255 all the magnitudes that are above a certain threshold (it has been fixed to 80 ), while setting to 0 the others.

These networks are kept separated (Sobel and Roberts kernels) or merged together by MDC to derive the corre-
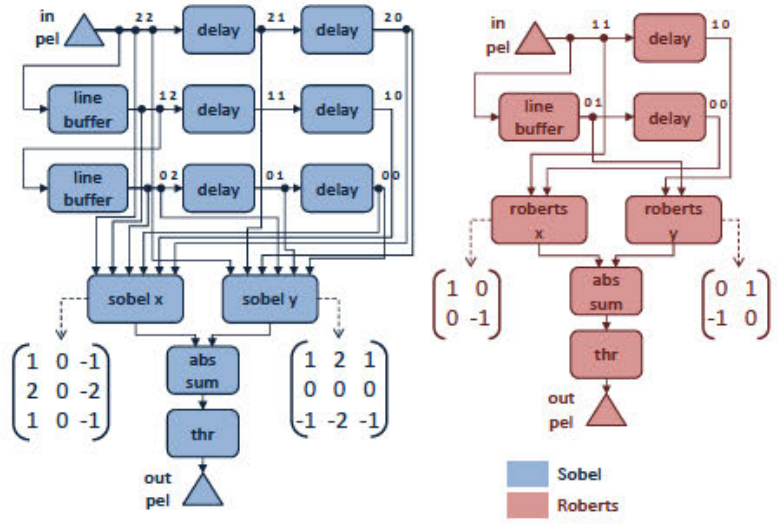

Fig. 5. Simplified dataflow graphs implementing the Sobel and the Roberts edge detection computational kernels.

sponding reconfigurable multi-dataflow model (reconfigurable kernel). The convolution actors (sobel $x$, sobel y, roberts $x$ and roberts_y) are not shareable, since they correspond to the different convolution kernel matrices applied on the input image. In this case, the actors that can be re-used between the two networks are related to the magnitude computation step (line buffer, delay and abs sum) and to the thresholding step (thr). In summary, Sobel and Roberts kernels involve respectively 14 and 7 actors (two more than the simplified version in Figure 5), while reconfigurable kernel has 14 SBoxes and 18 overall actors, among which 5 are shared between the Sobel and Roberts datapaths, and 13 not.

On the bases of the described Sobel, Roberts and reconfigurable kernels, three different designs are considered:

- fine-grain - a standard DPR-based ARTICo ${ }^{3}$ architecture where Sobel and Roberts kernels can be freely instantiated within a number of slots going from 1 to 4 .

- coarse-grain - an MDC-compliant reconfigurable accelerator built around the reconfigurable computational kernel, able to switch between Sobel and Roberts algorithms. Contrarily to ARTICo ${ }^{3}$, MDC cannot tune the number of accelerators to be used in parallel at runtime, so that a worst case configuration with 4 accelerators in parallel is adopted. - multi-grain - following the approach proposed in this work, this implementation is composed of heterogeneous reconfigurable MDC-generated reconfigurable kernels instantiated within the slots of the ARTICo ${ }^{3}$ architecture. The number of slots ranges from 1 to 4 .

Designs are labeled according to the granularity level of the reconfiguration behind them (e.g. fine is used when single bit connections are changed). Nevertheless, please note that both ARTICo $^{3}$ and MDC cores act on coarse areas of the FPGA. $\mathrm{ARTICo}^{3}$ slots, from a chip occupation perspective, are larger than MDC multi-flow datapaths, but they are reconfigured at bit level using DPR, which is why the standalone ARTICo ${ }^{3}$ designs are labeled as fine-grain. 
TABLE I

EXPERIMENTAL RESULTS FOR ALL THE CONFIGURATIONS OF THE CONSIDERED DESIGNS. *IN THE FORM OF $N k k$, WHERE $N$ IS THE NUMBER OF PARALLEL ACCELERATORS (SLOTS) AND $k$ IS THE KIND OF COMPUTATIONAL KERNEL OF EACH ACCELERATOR: FIXED Sobel (FS) AND Roberts (FR), OR reconfigurable EXECUTING SOBEL (RS) OR ROBERTS (RR) ALGORITHM (E.G. $4 f s$ IS THE FIXED, MEANT AS NOT RECONFIGURABLE, SOBEL PARALLELIZED IN 4 SLOTS; WHILE $4 r r$ IS THE RECONFIGURABLE ACCELERATOR, COMPUTING ROBERTS FUNCTIONALITY, PARALLELIZED IN 4 SLOTS). **COMING FROM REAL ON-BOARD POWER MEASUREMENTS. DATA IN BRACKETS SHOW THE PERCENTAGE VARIATION OF OF THE CONFIGURATIONS WITH RESPECT TO THE CASE WHERE ONLY ONE SLOT IS EXPLOITED.

\begin{tabular}{|c|c|c|c|c|c|c|c|c|}
\hline \multirow{2}{*}{ config* } & \multicolumn{4}{|c|}{ fps (for different image sizes) } & \multicolumn{4}{|c|}{ energy $* *[\mathrm{~mJ}]$ (for different image sizes) } \\
\hline & $256 \times 256$ & $512 \times 512$ & $1024 \times 1024$ & $2048 \times 2048$ & $256 \times 256$ & $512 \times 512$ & $1024 \times 1024$ & $2048 \times 2048$ \\
\hline \multicolumn{9}{|c|}{ fine-grain } \\
\hline Ifs & 26.95 & 6.61 & 1.65 & 0.42 & 33.47 & 130.32 & 532.13 & 2132.95 \\
\hline $2 f_{s}$ & $33.35(+24 \%)$ & $8.31(+26 \%)$ & $2.11(+28 \%)$ & $0.53(+25 \%)$ & $28.61(-14 \%)$ & $107.27(-17 \%)$ & $437.37(-17 \%)$ & $1756.8(-17 \%)$ \\
\hline $3 f_{s}$ & $34.57(+28 \%)$ & $9.01(+36 \%)$ & $2.26(+37 \%)$ & $0.57(+34 \%)$ & $26.76(-20 \%)$ & $102.38(-21 \%)$ & $406.44(-23 \%)$ & $1628.57(-23 \%)$ \\
\hline $4 f_{s}$ & $36.96(+37 \%)$ & $9.50(+44 \%)$ & $2.39(+44 \%)$ & $0.59(+40 \%)$ & $25.26(-24 \%)$ & $99.01(-24 \%)$ & $397.11(-25 \%)$ & $1562.94(-26 \%)$ \\
\hline $1 \mathrm{fr}$ & 26.91 & 6.64 & 1.70 & 0.42 & 32.36 & 131.76 & 526.17 & 2106.31 \\
\hline $2 f r$ & $33.48(+24 \%)$ & $8.43(+27 \%)$ & $2.12(+25 \%)$ & $0.53(+25 \%)$ & $27.23(-15 \%)$ & $106.58(-19 \%)$ & $429.57(-18 \%)$ & $1729.04(-17 \%)$ \\
\hline $3 f r$ & $34.58(+29 \%)$ & $9.00(+35 \%)$ & $2.28(+34 \%)$ & $0.57(+35 \%)$ & $26.93(-16 \%)$ & $100.42(-23 \%)$ & $402.62(-23 \%)$ & $1603.06(-23 \%)$ \\
\hline $4 f r$ & $35.98(+34 \%)$ & $9.42(+42 \%)$ & $2.37(+40 \%)$ & $0.60(+42 \%)$ & $25.29(-21 \%)$ & $96.08(-27 \%)$ & $387.19(-26 \%)$ & $1537(-27 \%)$ \\
\hline \multicolumn{9}{|c|}{ coarse-grain } \\
\hline $4 r s$ & 36.90 & 9.52 & 2.39 & 0.60 & 28.44 & 97.89 & 397.4 & 1578.09 \\
\hline $4 r r$ & 36.96 & 9.52 & 2.40 & 0.60 & 25.42 & 97.22 & 399.73 & 1570.45 \\
\hline \multicolumn{9}{|c|}{ multi-grain } \\
\hline $1 r s$ & 26.85 & 6.74 & 1.69 & 0.42 & 33.04 & 133.66 & 529.74 & 2136.26 \\
\hline $2 r s$ & $33.19(+24 \%)$ & $8.38(+24 \%)$ & $2.12(+25 \%)$ & $0.53(+25 \%)$ & $26.98(-18 \%)$ & $109.63(-17 \%)$ & $440.38(-16 \%)$ & $1751.99(-17 \%)$ \\
\hline $3 r s$ & $34.45(+28 \%)$ & $8.96(+33 \%)$ & $2.23(+32 \%)$ & $0.57(+35 \%)$ & $27.82(-15 \%)$ & $102.26(-23 \%)$ & $412.56(-22 \%)$ & $1636.79(-23 \%)$ \\
\hline $4 r s$ & $36.90(+37 \%)$ & $9.52(+41 \%)$ & $2.39(+41 \%)$ & $0.60(+42 \%)$ & $28.44(-13 \%)$ & $97.89(-26 \%)$ & $397.4(-24 \%)$ & $1578.09(-26 \%)$ \\
\hline $1 \mathrm{rr}$ & 26.90 & 6.62 & 1.70 & 0.42 & 32.11 & 131.02 & 527.9 & 2128.85 \\
\hline $2 r r$ & $33.06(+23 \%)$ & $8.42(+27 \%)$ & $2.12(+24 \%)$ & $0.53(+25 \%)$ & $27.01(-15 \%)$ & $107.24(-18 \%)$ & $435.99(-17 \%)$ & $1755.22(-17 \%)$ \\
\hline $3 r r$ & $34.37(+28 \%)$ & $8.10(+36 \%)$ & $2.28(+34 \%)$ & $0.57(+34 \%)$ & $26.36(-17 \%)$ & $103.15(-21 \%)$ & $406.78(-22 \%)$ & $1631.55(-23 \%)$ \\
\hline $4 r r$ & $36.96(+37 \%)$ & $9.52(+44 \%)$ & $2.40(+41 \%)$ & $0.60(+42 \%)$ & $25.42(-20 \%)$ & $97.22(-25 \%)$ & $399.73(-24 \%)$ & $1570.45(-26 \%)$ \\
\hline
\end{tabular}

\section{Experimental Results}

To evaluate the proposed approach, experimental results obtained with the considered designs under test are hereafter discussed. The target device for all the reported data is the Xilinx XC7Z020CLG484-1 available on the custom Zynq7000 board used, and the operating frequency has been set to $100 \mathrm{MHz}$. Note that energy numbers come from real on-board power measurements during execution. For the evaluation of the designs, several metrics will be taken into account:

- LUT, FF - resource occupancy of the design (for all its possible configurations) within the targeted device;

- fps - frames per second of the design (for all its possible configurations) while processing images;

- $E$ - energy consumption of the design (for all its possible configurations) while processing images;

- Reconfiguration overhead, evaluated in terms of:

- $B$ - memory footprint of the reconfiguration data;

- $T$ - time required for the reconfiguration phase;

- $E$ - energy consumption during the reconfiguration phase;

- $F$ - penalty on the achievable maximum operating frequency when $\mathrm{CG}$ reconfiguration is provided;

1) Processing Evaluation: Table I depicts hardware processing results for each possible configuration of the different designs. In terms of processing time, it shows resulting fps for different image sizes and for different degrees in terms of parallelism. With respect to the parallelism, both fine-grain and multi-grain designs show the same trend, increasing the processed fps up to $40 \%$ going from 1 to 4 slots. No appreciable difference between Sobel and Roberts kernel execution in terms of fps seems to be present. Note also that performance does not increase linearly, as it would be expected. The main reason behind this is the communication overhead introduced by the $\mathrm{ARTICo}^{3}$ architecture, and its predominance with respect to the real computing time (less than $10 \%$ of the overall time per block). To comply with the required dataparallel execution model, the image processing application requires partitioning the total workload in a given set of dataindependent sub-workloads that are executed in rounds. The size of the local workload has been fixed to process $32 \times 32$ image blocks, and the Runtime Library dispatches processing rounds in as many slots as available. Adopting more complex computational kernels or over-clocking data transfers could highlight differences between different kernels and executions with different number of slots.

Table I also shows the measured energy consumption for all configurations. As occurs for performance, the energy efficiency increases when using more accelerators (power consumption increase is less significant than the saving in terms of execution time), reaching reduction values of up to $27 \%$. Comparing the fine-grain and the multi-grain approaches, it is possible to see that energy consumption is similar. This means that, in an application scenario in which both edge detectors need to be present, the multi-grain accelerator provides a solution that is more energy efficient, since both datapaths are already available in the accelerator core logic (otherwise, two fine-grain accelerators would need to be present in the FPGA, or switched using DPR, which also adds an energy overhead as discussed in the following section). Note that, 
as for the fps metric, in terms of energy there is not a real difference between Sobel and Roberts kernel execution, meaning that CGR, in this very simple case, is not able to provide non-functional (fps/energy driven) adaptivity, but only a functional (i.e., algorithm-driven) one. The predominance of DMA transfers is also playing a role in terms of energy, flattening differences between the two kernels and among the different slot configurations.

2) Reconfiguration Granularity Analysis: Flexibility, as we already mentioned in the introduction, has a cost especially in terms of memory utilization, timing and consumed energy. Table II depicts overhead results of both fine-grain and coarsegrain reconfigurations, in terms of memory footprint (size $[\mathrm{B}]$ ), timing (time [ms]) and energy consumption (energy $[\mathrm{mJ}])$. As expected, the reconfiguration overhead of coarsegrain designs is much lower than the overhead of fine-grain ones. This is due to the fact that MDC-based reconfiguration requires writing a single configuration register for each accelerator to switch from one detector to another, while the DPR in $\mathrm{ARTICo}^{3}$ slots requires downloading a new bitstream for the whole region. In the presented scenario, the CG reconfiguration of four parallel accelerators requires around 1000 times less reconfiguration time (0.09 ms versus $106.14 \mathrm{~ms})$ and around 850 times less reconfiguration energy consumption $(0.11 \mathrm{~mJ}$ versus $94.11 \mathrm{~mJ})$ with respect to $\mathrm{FG}$ reconfiguration of four slots. The price to be paid for this advantage in terms of timing and energy is that coarse-grain designs are less flexible. It is possible to switch among the kernels to be executed, which are already implemented on the system, by simply multiplexing them in time. However, an infrastructure providing only CGR does not allow playing with the degree of parallelism or re-writing completely the functionality with a bitstream not originally considered at design time.

A multi-grain adaptive design combines the best of both, offering a fast low-power consuming reconfiguration when changing among functionalities is needed, and the performance trade-off (throughput versus energy consumption) given by the flexible parallelism offered by the DPR-based ARTICo ${ }^{3}$ architecture. In such a way, for the considered proof of concept involving Sobel and Roberts algorithms, MDC-based reconfiguration can be exploited to change the functionality of each slot in a lightweight manner, while the DPR approach behind $\mathrm{ARTICo}^{3}$ takes care of fps/energy by playing with the number of accelerators.

TABLE II

RECONFIGURATION OVERHEAD. * $N$ IS THE NUMBER OF PARALLEL ACCELERATORS (SLOTS). **REAL ON-BOARD POWER MEASUREMENTS

\begin{tabular}{rc|c|c|c} 
design & config* & size $[\mathbf{B}]$ & time $[\mathbf{m s}]$ & energy $^{* *}[\mathbf{m J}]$ \\
\hline \hline fine-grain & 1 & $858 \mathrm{k}$ & 16.42 & 15.18 \\
fine-grain & 2 & $1715 \mathrm{k}$ & 47.62 & 41.91 \\
fine-grain & 3 & $2573 \mathrm{k}$ & 75.95 & 67.1 \\
fine-grain & 4 & $3430 \mathrm{k}$ & 106.14 & 94.11 \\
coarse-grain & 4 & 2 & 0.09 & 0.11
\end{tabular}

3) Impact of CGR: The coarse-grain reconfiguration, which is used both in coarse-grain and multi-grain designs of this experimental evaluation, has an implementation overhead. As seen in Section II-C, the MDC tool merges together the input dataflow applications, in this case the Sobel and Roberts models depicted in Figure 5, sharing the common actors and making them accessible by means of multiplexers (SBoxes) inserted in the crossroads of common paths of data. This means that, when configured for the execution of the Sobel or Roberts edge detector and with respect to the standalone Sobel or Roberts kernels, the reconfigurable kernel pays a resource overhead given by the fact that the non-shared actors (belonging to the detector that is not currently executed) plus the SBoxes need to be considered. This overhead is clearly visible in Table III, where Sobel and Roberts occupy respectively $10 \%$ and $55 \%$ less LUT and FF than the reconfigurable kernel. If the two isolated kernels are instantiated in parallel (see the Sobel+Roberts row in Table III), thus allowing runtime switching among detection algorithms (without any additional overhead), the reconfigurable kernel saves more than $20 \%$ of the same resources, meaning that sharing actors is convenient and that the SBoxes overhead is affordable, according to the considered metric. However, the insertion of the switching modules has also another drawback: it can negatively affect the operating frequency. SBoxes are fully combinatorial; therefore, their presence may lengthen the critical path of the system, thus lowering the highest achievable frequency. In this case, non-reconfigurable Sobel and Roberts kernels support respectively $5 \%$ and $57 \%$ higher operating frequency than the reconfigurable one. For the parallel Sobel+Roberts solution, if a worst-case frequency has to be selected (that is also the case of reconfigurable kernel), the maximum frequency estimated for Sobel need to be considered.

TABLE III

CG RECONFIGURATION OVERHEAD (AFFECTING coarse-grain AND multi-grain DESIGNS IN SECTION IV-A). IN BRACKETS PERCENTAGES OF VARIATION OF EACH METRIC WRT CG reconfigurable DESIGN.

\begin{tabular}{r|cc|c} 
kernel & \multicolumn{2}{|c|}{ resources (@100 MHz) } & Fmax [MHz] \\
& LUT & FF & \\
\hline reconfigurable & 2225 & 2360 & 108.39 \\
\hline Sobel & $1817(-18 \%)$ & $2076(-12 \%)$ & $113.92(+5 \%)$ \\
Roberts & $922(-59 \%)$ & $1048(-56 \%)$ & $170.13(+57 \%)$ \\
Sobel+Roberts & $2739(+23 \%)$ & $3124(+32 \%)$ & $113.92(+5 \%)$
\end{tabular}

4) Usage of the Proposed Flow: Previous discussions demonstrated the effectiveness of multi-grain reconfiguration, showing the advantage, in terms of timing and energy consumption, of mixing both $\mathrm{ARTICo}^{3}$ and MDC reconfiguration approaches. It is important to highlight also the effectiveness of the proposed flow in terms of design time and effort. The proposed toolchain speeds the design of multi-grain systems up by automatically mapping different input specifications in one MDC multi-flow datapath compliant with the DPR-Based $\mathrm{ARTICo}^{3}$ slots. Users only need to define the applications behavior through abstract high level input dataflow specifications; then, the toolchain takes care of the complete process from dataflow to multi-grain systems. Please, refer to [12], [14] for further evaluation of the single components of the 
integrated toolchain (e.g., automation level, user friendliness, stability, flexibility, etc.).

The effort of designing the dataflows is application-specific and cannot be evaluated. However, it is necessary to be highlighted, as the usage of dataflow specifications allows the exploitation of HLS dataflow-to-hardware tools (such as CAPH [23]), which not only speed the design process up by automating HDL generation, but also allow developers that are not expert in hardware design to adopt the proposed design flow.

Furthermore, the proposed toolchain facilitates the management of the generated multi-grain system, since the Runtime Library of the ARTICo ${ }^{3}$ architecture is capable of transparently managing the application execution and computation offloading to the hardware accelerators also when these are CGR accelerators.

\section{Conclusions}

In this paper, a multi-grain reconfiguration approach that relies on both coarse-grain (time-multiplexing of available datapaths) and fine-grain (time-multiplexing of FPGA resources) reconfiguration mechanisms has been proposed. The combination of these mechanisms, eased by the use of $\mathrm{ARTICo}^{3}$ and MDC, can be used to enable advanced functional and nonfunctional adaptivity support in CPS. The presented toolchain supports the automatic development, from specification down to implementation, of multi-grain reconfigurable systems, speeding up the design process and facilitating their deployment and run-time management.

A proof of concept test case involving two edge detection kernels has been used to evaluate the proposed reconfiguration infrastructure. Experimental results demonstrate the potential of the approach in terms of FPGA resources, timing and energy efficiency. Drawbacks and strengths of the different reconfiguration granularities have been highlighted, and the advantages of leveraging on a multi-grain reconfigurable architecture have been revealed. The proposed methodology can be particularly useful in CPS contexts, where variability is common due to the involvement of user (e.g. image resolution), environment (e.g. speed of the objects whose edges have to be detected) or system (e.g. remnant battery) requirements.

With the proposed proof of concept, a limited set of the adaptivity potential for the proposed approach has been shown. In the considered scenario, lightweight MDC-based adaptation provides functional adaptation but, in cases where the implemented computational kernels differ substantially, it can be used to provide also non-functional adaptation with performance/energy tradeoffs [3]. Similarly, ARTICo ${ }^{3}$-based adaptivity could achieve better results with scalability for more complex applications or could provide fault tolerance [12]. In the future, a deeper validation of the proposed architecture is to be performed by adopting a larger set of applications and/or more complex ones, such as Gaussian edge detectors like Canny, or by considering error-affected scenarios and approximate computing.

\section{ACKNOWLEDGMENT}

This work has received funding from the European Union's Horizon 2020 research and innovation programme under grant agreement No 732105. The authors would like to thank the Spanish Ministry of Education, Culture and Sport for its support under the FPU grant program. Leonardo Suriano holds a predoctoral contract under RR01/2015 (Programa Propio) by Universidad Politécnica de Madrid.

\section{REFERENCES}

[1] Xilinx, Vivado Design Suite User Guide - Partial Reconfiguration UG909 (v2017.1), 2017.

[2] M. Lombardo et al., "Power management techniques in an FPGA-based WSN node for high performance applications," in Int. Workshop on Reconfigurable and Communication-Centric Systems-on-Chip (ReCoSoC), pp. 1-8, July 2012.

[3] C. Sau et al., "Challenging the Best HEVC Fractional Pixel FPGA Interpolators with Reconfigurable and Multi-frequency Approximate Computing," IEEE Embedded Systems Letters, vol. 9, no. 3, pp. 6568, 2017.

[4] R. Hartenstein, "Coarse grain reconfigurable architecture (embedded tutorial)," in Proc. of the Asia and South Pacific Design Automation Conf., 2001

[5] K. Paul et al., "reMORPH: A Runtime Reconfigurable Architecture," in Digital System Design (DSD), 2012 15th Euromicro Conference on, pp. 26-33, September 2012.

[6] Z. Rakossy et al., "Design and analysis of layered coarse-grained reconfigurable architecture," in Int. Conf. on Reconfigurable Computing and FPGAs, pp. 1-6, December 2012.

[7] "RPCT project. http://sites.unica.it/rpct/."

[8] M. Amagasaki et al., "An Embedded Reconfigurable IP Core with Variable Grain Logic Cell Architecture," Int. J. Reconfig. Comp., vol. 2008, pp. 180216:1-180216:14, 2008.

[9] A. Thomas et al., "HoneyComb: An Application-Driven Online Adaptive Reconfigurable Hardware Architecture," Int. J. Reconfig. Comp., vol. 2012, p. 17 pages, 2012

[10] I. Sourdis et al., "DeSyRe: On-demand system reliability," Microprocessors and Microsystems - Embedded Hardware Design, vol. 37, no. 8, pp. 981-1001, 2013.

[11] F. L. Yuan et al., "A Multi-Granularity FPGA With Hierarchical Interconnects for Efficient and Flexible Mobile Computing," IEEE Journal of Solid-State Circuits, vol. 50, pp. 137-149, Jan 2015.

[12] A. Rodríguez et al., "FPGA-Based High-Performance Embedded Systems for Adaptive Edge Computing in Cyber-Physical Systems: The ARTICo $^{3}$ Framework," Sensors, vol. 18, no. 6, 2018.

[13] E. Lee and T. Parks, "Readings in Hardware/Software Co-design," pp. 59-85, Kluwer Academic Publishers, 2002.

[14] F. Palumbo et al., "Power-Awarness in Coarse-Grained Reconfigurable Multi-Functional Architectures: a Dataflow Based Strategy," Journal of Signal Processing Systems, vol. 87, pp. 81-106, Apr 2017.

[15] C. Sau et al., "Reconfigurable coprocessors synthesis in the MPEG-RVC domain," in Int. Conf. on ReConFigurable Computing and FPGAs, 2015.

[16] E. Davies, "Circularity a new principle underlying the design of accurate edge orientation operators," Image and Vision Computing, vol. 2, no. 3 , pp. 134-142, 1984.

[17] S. Zhao et al., "Sobel-LBP," in 2008 15th IEEE International Conference on Image Processing, pp. 2144-2147, Oct 2008.

[18] L. Roberts et al., "Machine perception of three dimensional solids, in Optical and Electro-Optical Information Processing," MIT press, pp. 159-197, 1965.

[19] G. Chaple et al., "Comparisons of Robert, Prewitt, Sobel operator based edge detection methods for real time uses on FPGA," in Int. Conf. on Technologies for Sustainable Development (ICTSD), 2015.

[20] J. Burnham et al., "Comparison of the Roberts, Sobel, Robinson, Canny, and Hough Image Detection Algorithms," in MS State DSP Conf., 1997.

[21] S. Singh and B. Singh, "Effects of noise on various edge detection techniques," in Int. Conf. on Computing for Sustainable Global Development (INDIACom), March 2015.

[22] W. Yang et al., "Recognizing and tracking airport runway target in infrared images," in Proc. National Aerospace and Electronics Conf. NAECON, 1997.

[23] J. Sérot et al., "CAPH: a language for implementing stream-processing applications on FPGAs," in Embedded Systems Design with FPGAs, Springer, 2013. 